\title{
Non vi sono diritti senza doveri
}

\author{
di Elio Borgonovi
}

L'approvazione dei nuovi LEA ripropone un tema fondamentale per ogni servizio sanitario nazionale, ossia quello del diritto alla tutela della salute. In Italia tale diritto è riconosciuto costituzionalmente dall'articolo 32, che recita: $<L a$ Repubblica tutela la salute come fondamentale diritto dell'individuo e interesse della collettività, e garantisce cure gratuite agli indigenti. Nessuno può essere obbligato a un determinato trattamento sanitario se non per disposizione di legge. La legge non può in nessun caso violare i limiti imposti dal rispetto della persona umana».

Questa dichiarazione comprende alcuni principi che meritano di essere chiariti: la tutela della salute come fondamentale diritto dell'individuo, l'interesse della collettività, le cure gratuite agli indigenti, la libertà dell'individuo che non può essere obbligato a trattamenti sanitari (se non per obblighi di legge), il rispetto della persona umana. Innanzitutto, va chiarito che la tutela della salute non deve essere confusa con il diritto alla salute. Il progresso scientifico e una errata interpretazione del positivismo scientista hanno infatti contribuito a diffondere una idea di "quasi immortalità", idea che si sta rafforzando anche con la possibilità di realizzare organi artificiali. A parte le considerazioni di ordine filosofico, etico e religioso, va sottolineato che questa errata interpretazione contribuisce a generare in molti un errato rapporto con la malattia, la sofferenza e perfino con la morte. Un rapporto che ha riflessi di carattere organizzativo, economico e relazionale. Sul piano organizzativo si rileva che in molte persone crescono in modo abnorme le aspettative nei confronti delle aziende sanitarie e delle aziende ospedaliere. Questi atteggiamenti hanno riflessi rilevanti sul piano economico, poiché costituiscono una delle cause che determinano la medicina difensiva, e sul piano relazionale, in quanto si indeboliscono i rapporti di fiducia con il medico, gli infermieri, gli altri operatori sanitari e socio-sanitari.

Il concetto di diritto fondamentale dell'individuo spesso viene confuso con diritto assoluto. Ciò rende difficile definire priorità, non solo per chi deve compiere scelte di politica sanitaria, ma anche per chi deve compiere scelte manageriali di allocazione di risorse limitate e per chi deve compiere scelte di carattere professionale. Le persone e le istituzioni che hanno la responsabilità di erogare prestazioni e servizi di tutela della salute sono così sottoposte a pressioni che è sempre più difficile gestire. Se è vero che ognuno di noi percepisce prima di tutto il proprio bisogno di salute, è altrettanto vero che i diritti di ogni individuo e di ogni gruppo sociale (es. pazienti affetti da determinate patologie) devono essere contemperati con i diritti di altri individui, gruppi sociali e/o pazienti. Se nella società non si diffonde anche con riguardo al tema della salute il senso di responsabilità, sarà sempre più difficile ricercare e trovare soluzioni in grado di definire le priorità in rapporto alla severità della condizione di salute. In assenza di senso di responsabilità e di autoregolazione delle attese di ciascuno con riguardo al proprio stato di salute, il rischio è quello di privilegiare la tutela di bisogni di salute meno severi ma che riguardano un elevato numero di persone (per ottenere consenso politico) o di dare priorità a quelle malattie molto gravi, molto rare e a elevato costo di trattamento che trovano eco nell'opinione pubblica, trascurando altre malattie altrettanto gravi, rare e costose che, per esempio, non hanno trovato testimonial ad alta visibilità. Peraltro queste considerazioni hanno una rilevanza sul piano economico, in quanto una più elevata coscienza dei propri diritti se confrontata con $i$ diritti di altri potrebbe rendere più accettabili forme di 
compartecipazione ai costi (ticket) da parte di coloro che economicamente sono in grado di sostenere tale onere. Ovviamente per diffondere questo senso di responsabilità è necessaria anche una corretta informazione, nel senso che la compartecipazione ai costi (ticket) non dovrebbe essere presentata come modalità per raccogliere maggiori entrate ma come strumento per "dare a ognuno il necessario quando si è in condizione di risorse limitate".

Il terzo elemento sottolineato nell'articolo 32 è quello della tutela della salute come interesse della collettività. Ciò richiama non solo l'importanza della "sanità pubblica" (es. vaccinazioni, prevenzione contro epidemie e altre malattie trasmissibili, controllo del rapporto tra salute umana e salute animale) ma anche la centralità della persona in senso umanistico. La tutela della salute non è solo dettata dalle ragioni di conservazione del "capitale umano", concetto, quest'ultimo, che non deve essere inteso solo in senso produttivistico ed economico, in quanto persone in buona salute contribuiscono ad aumentare il prodotto interno lordo, ma in senso di contributo che persone in buona salute possono dare al sistema delle relazioni umane. Per esempio efficaci modelli assistenziali per persone con patologie croniche, con disabilità, non autosufficienti, contribuiscono a una migliore qualità di vita delle famiglie. Purtroppo la carenza o l'inadeguatezza di servizi di tutela della salute, soprattutto per queste condizioni di salute, limitano enormemente la possibilità delle famiglie di avere una propria vita indipendente e spesso sono causa di drammi familiari (omicidisuicidi) o drammi di persone che si sentono sole e abbandonate. L'indebolimento dell'attenzione all'interesse generale è presente anche in comportamenti che recentemente si sono diffusi nel nostro Paese, come quello contro le vaccinazioni, atteggiamenti che sono dettati dal prevalere di presunti interessi individuali (evitare rischi collegati alle vaccinazioni, che peraltro dal mondo scientifico sono considerati estremamente limitati) rispetto all'interesse generale, poiché la caduta del tasso di vaccinazione al di sotto dell' $85 \%$ comporta rischi per la popolazione.

Il quarto elemento costitutivo è espresso nella parte di articolo 32 nel quale si afferma «la Repubblica [... ] garantisce cure gratuite agli indigenti». Questa parte deve ritenersi formalmente superata con l'introduzione del SSN con la Legge 833/78, nella quale è applicato il principio di universalità della tutela della salute, nonché dal sistema delle "esenzioni alla compartecipazione" previsto per le persone con basso reddito. Tuttavia, sul piano sostanziale, il problema non appare completamente risolto, in quanto di fronte a livelli essenziali di assistenza uniformi in tutto il Paese si hanno poi livelli effettivi di assistenza assai differenziati. Appare superfluo ricordare i numerosi studi, spesso citati anche in Mecosan, relativi alle differenze nella qualità e quantità delle prestazioni dei servizi erogati nelle regioni italiane e in diverse parti della stessa regione. Ciò lascia aperta per coloro che intendono difendere il modello di SSN la sfida del superamento delle differenze di tutela effettiva. Una sfida che va affrontata con riferimento alla componente di solidarietà che non è solamente di tipo economico, che tipicamente si realizza agendo sui criteri di finanziamento dallo Stato alle Regioni, ma è anche di tipo manageriale (diffusione delle capacità di gestione delle Aziende Sanitarie e delle Aziende Ospedaliere), professionale (trattare nello stesso modo pazienti del SSN e pazienti che possono permettersi di pagare prestazioni intramoenia), organizzativo (tener conto che ai bisogni delle persone indigenti si risponde con servizi sanitari, servizi socio-sanitari e servizi socio-assistenziali tra loro integrati) e culturale (spesso le persone indigenti e con bisogni più significativi sono anche quelle meno informate sui propri diritti). L'applicazione dell'articolo 32 richiede di agire contestualmente su queste leve e significa anche affrontare in modo non ideologico il problema della sostenibilità economica del SSN. Il diritto alla salute delle persone indigenti, meno informate e perciò con una minore accessibilità ai servizi del SSN non si difende né sostenendo la gratuità delle prestazioni per tutti, né teorizzando il passaggio alle "regole del mercato" per coloro che possono permetterselo economicamente. Si difende tramite il rafforzamento della solidarietà generale garantita dal SSN finanziato con i tributi, con la solidarietà volontaria consentita da fondi negoziali, fondi e casse aziendali (il cosiddetto "secondo pilastro del welfa$r e^{\prime \prime}$ e con responsabili scelte individuali di chi decide di destinare parte del proprio reddito alla copertura di rischi di salute, invece che ad altri bisogni (assicurazioni private). Si tratta di un sistema ben diverso, per esempio, da quello degli USA che si basa non sul principio di solidarietà collettiva ma sulla preminenza del principio di scelta individualistica (assicurazioni private), integrato con interventi pubblici per alcuni gruppi della popolazione (MedicAid, MediCare e altri programmi pubblici minori) e, recentemente, con l'Affordable Care Act (riforma Obama) che per ora il Presidente Trump non è riuscito a cancellare. 
L'ultima parte dell'articolo 32 della Costituzione Italiana si caratterizza per l'equilibrio tra esigenze dell'individuo e interesse della collettività. Infatti, solo la Legge può imporre trattamenti obbligatori, consentiti esclusivamente quando si manifestano esigenze di interesse generale, compreso quello di tutela dell' individuo quando vi è il concreto rischio di comportamenti autolesionisti. Peraltro viene enunciato anche un limite alla Legge, che discende dal concetto di diritto naturale, nel senso che non possono essere imposti trattamenti obbligatori contrari alla dignità umana. Si tratta di un equilibrio di principi che, oltre ad avere un valore di tipo etico, morale e legale, ha implicazioni molto concrete di fronte al problema della sostenibilità economica. In alcuni Paesi già oggi si pone un limite all'erogazione di servizi per ragioni economiche, per esempio non viene garantita la dialisi per persone con età superiore ai 65 anni, o si minaccia di non garantire l'assistenza a persone che hanno abitudini contrarie alla salute (es. patologie conseguenti a obesità, fumo, dipendenze ecc.). Ma all'orizzonte si prospettano alcune ipotesi che porranno rilevanti problematiche di carattere etico. Con le possibilità offerte dalla genomica e dalla medicina predittiva non solo vi sarà per gli individui la possibilità di chiedere interventi finalizzati a evitare l'insorgere di malattie, ma potrebbe nascere nelle autorità pubbliche la tentazione di sottoporre alcune persone a interventi preventivi finalizzati a ridurre costi futuri. Per evitare queste inquietanti prospettive occorre agire sulla cultura e sul continuo richiamo al rispetto della dignità della persona, che non può essere subordinata in alcun modo a considerazioni di tipo economico. Per i diritti la cui esigibilità dipende dalla disponibilità di risorse, si devono trovare condizioni di compatibilità, il che non vuol dire assolutamente subordinazione del diritto alle ragioni dell'equilibrio economico.

In effetti si hanno livelli di compatibilità, in termini quantitativi e qualitativi, più o meno soddisfacenti in rapporto all'accettazione da parte dei diversi soggetti del principio di equilibrio tra diritti e doveri. I pazienti hanno diritto alla tutela della salute ma devono sentire anche il dovere di contribuire alla propria, per esempio con stili di vita favorevoli alla prevenzione, diagnosi precoce, compliance nel seguire le prescrizioni di medici, corretto uso di farmaci/protesi senza causare sprechi al SSN, attivazione di tutte le proprie energie per affrontare situazioni di difficoltà (al riguardo si possono citare i tanti esempi di persone con disabilità o invalidità anche gravi che riescono a svolgere autonomamente molte attività). Medici, infermieri e altri professionisti della salute hanno il diritto di vedere riconosciuta la propria professionalità anche sul piano economico e di trovarsi in organizzazioni che consentono loro di esprimersi al meglio, ma hanno il dovere di porre il paziente nella sua completezza al centro delle proprie scelte e del proprio operare. Non devono pensare di occuparsi di malattie, organi, o di garantire semplicemente prestazioni, ma devono agire riflettendo sempre sul fatto che si trovano di fronte a persone che vanno considerate e rispettate in quanto tali. I manager hanno il dovere di realizzare le migliori condizioni organizzative per i pazienti e per i professionisti e di applicare soluzioni idonee a ridurre i vincoli derivanti dalla limitatezza delle risorse per bilanciare il diritto di dirigere e coordinare il lavoro di altri e di perseguire l'equilibrio di bilancio. Coloro che decidono le politiche di tutela della salute, siano essi i responsabili politici o i dirigenti di Regioni e del Ministero della Salute, devono sentire il dovere di definire priorità sulla base di una conoscenza della realtà e di un attento ascolto delle esigenze dei pazienti, come bilanciamento del ancora diritto giuridicamente riconosciuto di definire "vincoli compatibili con la limitatezza delle risorse" come si usa dire.

Mentre sul piano giuridico è doveroso parlare di equilibrio tra diritti e doveri, sul piano dei comportamenti delle persone è opportuno parlare di equilibrio tra scienza e coscienza, tra razionalità (tecnica, organizzativa, economica) ed etica (richiamo ai valori fondamentali). Scienza poiché è richiesto un continuo impegno a migliorare le conoscenze, competenze e capacità riguardanti la tutela della salute, ad aggiornarsi, ad affinare la propria professionalità con l'esperienza, ad applicare la cultura del merito. Coscienza poiché ognuno è chiamato ad attuare scelte difficili guidate dal principio del rispetto della persona come individuo e dei valori su cui poggia una società che si vuole dire civile. Infatti, il progresso della scienza contribuirà a spostare l'orizzonte delle scelte sempre più sulla frontiera del rapporto tra vita e morte, sofferenza e accettazione/rifiuto della stessa, rispetto della libertà di scelta e della dignità della persona. Queste problematiche sono state sempre presenti nella storia dell'assistenza, negli ultimi 20-30 anni si è ritenuto (si è avuta la presunzione) di poterle superare richiamandosi alla razionalità dei comportamenti ma sono destinate a riproporsi nella loro drammatica attualità con la medicina personalizzata (rischio che il diritto di 
pochi vada a scapito del diritto di molti), di precisione (rischio che con interventi di chirurgia del cervello o con farmaci a elevata efficacia si possa influenzare la volontà dei singoli), predittiva (rischio che si generi l'illusione di sottrarsi alla sofferenza) e partecipativa (rischio che il coinvolgimento del paziente comporti la deresponsabilizzazione di medici, infermieri e altri professionisti).

La scienza consente di alzare continuamente l'asticella in termini di complessità dei problemi di salute cui è possibile dare risposte efficaci, la razionalità consente di affrontare i problemi di sostenibilità, ma solo coscienze guidate da un forte senso etico consentono un vero progresso civile e umano. Nella società moderna molti cercano la sicurezza nella scienza e nella razionalità, propongono soluzioni ottimali per affrontare i temi della sostenibilità tecnica, organizzativa ed economica, ritenendo con ciò di poter ridurre il ruolo della coscienza e dell'etica. Ai lettori di Mecosan si propone invece una vita "meno tranquilla e più affascinante", poiché di fronte all' insondabilità dell'esistenza, della sofferenza e della morte solo coscienze fondate su un forte senso etico possono aiutare a utilizzare al meglio $i$ benefici prodotti dalla scienza. 